rheuma plus 2014 $\cdot 13: 1$

DOI 10.1007/s12688-014-0010-2

Online publiziert: 14. November 2014

○) Springer-Verlag Wien 2014

Burkhard Leeb

II. Med. Abteilung, NOE Kompetenzzentrum für Rheumatologie, Stockerau, Österreich

\title{
Verbesserte Therapiemöglichkeiten
}

gen zur Schwangerschaft bei SLE-Patientinnen.

War früher dieses Problem nicht wirklich vorhanden, ist es heutzutage, durch die verbesserten therapeutischen Möglichkeiten immer ein Thema, das praktizierende RheumatologInnen beschäftigt.

Ein eigener Beitrag der Rubrik „Aktuell“ widmet sich der analgetischen Wirkung von Trainingstherapie und leitet damit über zu der bemerkenswerten Übersichtsarbeit von Paul Haber über Trainingstherapie bei rheumatoider Arthritis. Auch dieser Aspekt gewinnt mit verbesserten Therapiemöglichkeiten für die Betroffenen immer mehr an Bedeutung. Wer bewegt sich schon gerne unter Schmerzen? Daher wird es eine wesentliche Aufgabe für uns sein, Überzeugungsarbeit unter den PatientInnen zu leisten und die Bedeutung der Trainingstherapie für die Prognose bekannt zu machen. Motto etwa: „Wer trainiert, hat weniger Schmerzen und lebt besser."

Manfred Herold, wohl einer der profundesten Kenner des Gasteiner Stollens, beschäftigt sich in seiner Übersicht mit dieser wohl einzigartigen Therapieoption für PatientInnen mit seronegativer Spondarthritis. Die genauen Mechanismen sind nach wie vor nicht komplett bekannt; Tatsache ist aber, dass viele PatientInnen deutlich von den Stolleneinfahrten profitieren und damit z. B. NSAR einsparen können.

Klemens Rappersberger und Leo Richter bringen uns die dermatologische Sicht der Arthritis psoriatica näher. Für uns Rheumatologen ist es zweifellos von groBer Bedeutung auch die Haut in die Überlegungen zum Management dieser zweithäufigsten destruktiven Gelenkentzündung einzubeziehen. Wir sollten doch eigentlich immer den Menschen und nicht nur einzelne Organsysteme behandeln. Dies kann das Management der Arthritis psoriatica komplizierter, aber auch herausfordernd und interessant machen.

Wie immer an dieser Stelle möchten wir Sie herzlich dazu einladen, uns Ihre Meinung zu den Beiträgen dieser Ausgabe, wie auch zu allen rheumatologischen Themen, die Sie für relevant oder für diskussionswert halten, zu schicken. Sie können in jedem Falle auf eine faire Review mit entsprechendem Feedback vertrauen.

Zum Abschluss möchte ich der Hoffnung Ausdruck geben, dass Sie in dieser Ausgabe für sich Interessantes finden werden. Wie immer, sage ich für Kritik, Hinweise, Zustimmung und für jeden anderen Beitrag auch schon im Voraus Danke.

$\mathrm{Zu}$ den bevorstehen Feiertagen und dem Jahreswechsel die besten Wünsche,

Herzlichst,

Ihr

Burkhard Leeb

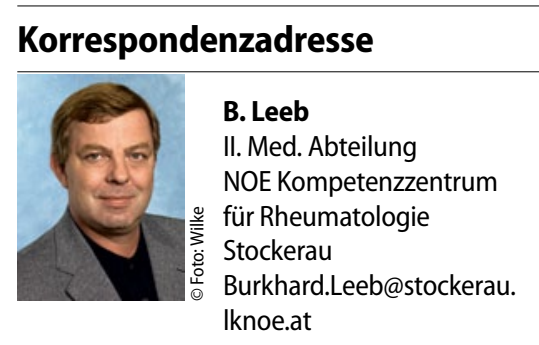

